\title{
Nonlinear Schrödinger equation on a circle
}

\author{
M. A. Smondyrev,${ }^{*}$ P. Vansant ${ }^{\dagger}$ F. M. Peeters ${ }^{\ddagger}{ }^{\ddagger}$ and J. T. Devreese ${ }^{\S}$ \\ Universitaire Instelling Antwerpen (UIA), Departement Natuurkunde, Universiteitsplein 1, B-2610 Wilrijk, Belgium
}

(Received 1 February 1995)

\begin{abstract}
The nonlinear Schrödinger equation is solved on an infinitesimal thin ring or circle. We obtained the exact real wave functions with their corresponding energies for the ground state and the excited states. Critical values of the circle perimeter are found at which the ground state changes its structure and additional higher excited states appear. Also, the complex wave functions that correspond to energy levels with finite angular momentum are studied.
\end{abstract}

\section{INTRODUCTION}

Mesoscopic systems have exhibited new interesting physics. In these systems interference effects are important. The one-dimensional ring system has attracted considerable experimental and theoretical attention. New effects have been discovered, e.g., discrete energy levels ${ }^{1}$ of closed systems and energy bands for a one-dimensional metal ring enclosing a magnetic flux (when observing the energy as a function of the flux) are found. The idea of persistent currents that are flux periodic in real normal-metal rings, was proposed in Refs. 2 and 3 for one-dimensional systems. An analogous system, e.g., an optical ring, has led to several applications, such as the laser gyroscope ${ }^{4}$ bistability effects in ring lasers, ${ }^{5}$ and optical band structures in passive ${ }^{6}$ and rotating ${ }^{7}$ ring resonators (see also Ref. 8).

Usually people deal with systems on a ring in the scope of linear physics. ${ }^{2}$ On the other hand there exist nonlinear phenomena described by the so-called nonlinear Schrödinger equation in which the potential depends on the wave function. This equation has been used (see Ref. 9 and references quoted herein) to describe stationary $2 \mathrm{D}$ self-focusing of a plane wave, self-trapping phenomena in nonlinear optics, propagation of heat pulses in solids, Langmuir waves in plasmas, etc. The equation is also related to the Ginzburg-Landau theory of superconductivity. Recently, we approached ${ }^{10}$ the same equation from the side of strongly coupled polaron physics, in which the polarons are confined in a potential box. The resulting effective nonlinear Schrödinger equation was solved exactly making use of elliptic functions. ${ }^{11-13}$

In the present paper we show that the $1 \mathrm{D}$ nonlinear Schrödinger equation (which was considered for the polaron problem in Refs. 14, 15, and 10) can also be solved in the case of periodic boundary conditions. The latter corresponds to a circle or, equivalently, an infinitesimal thin-ring system considering the strong electron-phonon coupling limit. The goal of the present paper is to show that former calculations for a ring with a magnetic flux perpendicular to this ring should be looked at carefully. From the solutions of the nonlinear Schrödinger equation for a circle or one-dimensional (1D) ring structure we can conclude that additional real solutions could play a role

in the description of the above system when including a magnetic field and, maybe, could provide an answer to some open questions which are raised for such systems. Here we will study how the nonlinear phenomena are correlated with the geometry of the system.

The paper is organized as follows. In Sec. II the real solutions to this equation are found. The complex solutions are given in Sec. III and our conclusions in Sec. IV.

\section{REAL SOLUTIONS}

\section{A. Basic equations and the trivial solution}

The Hamiltonian of a polaron confined in a onedimensional structure is of the form

$$
H=\frac{p^{2}}{2 m}+\sum_{k} \hbar \omega_{k} a_{k}^{\dagger} a_{k}+\sum_{k}\left[V_{k} a_{k} e^{i k z}+V_{k}^{*} a_{k}^{\dagger} e^{-i k z}\right]
$$

where $z$ and $p$ are the position and momentum operators of the electron, $m$ is the electron band mass, and $\omega_{k}$ is the frequency of the phonons with wave vector $k$. We include no external potential in the Hamiltonian (2.1), so we consider a medium without impurities.

As is well known, a system in the strong-coupling limit is described by the Schrödinger equation ${ }^{10}$

$$
-\frac{\hbar^{2}}{2 m} \frac{\partial^{2} \phi(z)}{\partial z^{2}}+V_{\mathrm{eff}}(z) \phi(z)=E \phi(z),
$$

with the effective potential

$V_{\text {eff }}(z)=\sum_{k}\left|V_{k}\right|^{2} \frac{\left|\rho_{k}\right|^{2}}{\hbar \omega_{k}}-\sum_{k} \frac{\left|V_{k}\right|^{2}}{\hbar \omega_{k}}\left[\rho_{k}^{*} e^{i k z}+\rho_{k} e^{-i k z}\right]$,

where $\rho_{k}=\int d z|\phi(z)|^{2} e^{i k z}$. In the case of an electron interacting with LO phonons we have $\omega_{k}=\omega_{\text {LO }}$ and

$$
V_{k}=-i \hbar \omega_{\mathrm{LO}}\left(\frac{2 \alpha^{\prime}}{L} \sqrt{\frac{\hbar}{2 m \omega_{\mathrm{LO}}}}\right)^{1 / 2}
$$


where $\alpha^{\prime}$ is the dimensionless electron-phonon coupling constant $^{16,17}$ and $L$ is the perimeter of the circle. Performing the scaling $z \rightarrow \lambda z$ with $\lambda=\sqrt{\hbar / m \omega_{\mathrm{LO}}} / \alpha^{\prime}$ we arrive at the nonlinear effective Schrödinger equation in dimensionless units:

$$
-\frac{1}{2} \frac{\partial^{2} \phi(z)}{\partial z^{2}}+V_{\mathrm{eff}}(z) \phi(z)=\varepsilon \phi(z),
$$

with $\varepsilon=E / \hbar \omega_{\mathrm{LO}} \alpha^{2}$ and

$$
V_{\mathrm{eff}}(z)=\sqrt{2} \int_{0}^{a} d z|\phi(z)|^{4}-2 \sqrt{2}|\phi(z)|^{2}
$$

where the perimeter of the circle can be written as $L=$ $\left(a / \alpha^{\prime}\right) \sqrt{\hbar / m \omega_{\text {LO }}}$ with $a$ the dimensionless perimeter.

Let us consider first the real wave functions. Equation (2.5) is of the form

$$
-\frac{1}{2} \phi^{\prime \prime}+\sqrt{2} B \phi-2 \sqrt{2} \phi^{3}-\varepsilon \phi=0,
$$

with $B=\int_{0}^{a} d z|\phi(z)|^{4}$. For the present case of a circle we have to invoke periodical boundary conditions

$$
\phi(0)=\phi(a), \quad \phi^{\prime}(0)=\phi^{\prime}(a) .
$$

First of all there exists the trivial solution (a constant wave function) $\phi(z)=1 / \sqrt{a}$, which is normalized on a circle. From Eq. (2.7) we obtain the corresponding energy level

$\varepsilon_{0}^{(0)}=-\frac{\sqrt{2}}{a} \quad$ or $\quad E_{0}^{(0)}=-\hbar \omega_{\mathrm{LO}} \frac{\sqrt{2} \alpha^{\prime}}{L} \sqrt{\frac{\hbar}{m \omega_{\mathrm{LO}}}}$.

To obtain the nontrivial solutions we notice that the first integral of Eq. (2.7) can be obtained readily (energy conservation)

$$
-\frac{1}{4} \phi^{\prime 2}+\frac{1}{\sqrt{2}} \phi^{2}\left(A-\phi^{2}\right)=C,
$$

where we used the notation $A=B-\varepsilon / \sqrt{2}$. In case of real wave functions Eq. (2.10) leads to the inequality $A^{2} \geq 4 \sqrt{2} C$. Therefore, we proceed by introducing two new parameters $\Phi_{1}$ and $\Phi_{2}$ by the relations $\sqrt{2} C=\Phi_{1} \Phi_{2}, A=\Phi_{1}+\Phi_{2}$. Because of symmetry $\Phi_{1}$ and $\Phi_{2}$ can be chosen in such a way that $\Phi_{2} \geq \Phi_{1}$. Equation (2.10) can now be written as

$$
\phi^{\prime 2}(z)=2 \sqrt{2}\left[\phi^{2}(z)-\Phi_{1}\right]\left[\Phi_{2}-\phi^{2}(z)\right] .
$$

\section{B. First set of solutions}

First, we consider $\Phi_{1} \geq 0$ and from Eq. (2.11) we immediately find that $0 \leq \Phi_{1} \leq \phi^{2}(z) \leq \Phi_{2}$. The integration of Eq. (2.11) gives us the general solution ${ }^{10}$

$$
\phi_{1}(z)=\sqrt{\Phi_{2}} \operatorname{dn}\left[2^{3 / 4} \sqrt{\Phi_{2}}\left(z-z_{0}\right) \mid m_{1}\right],
$$

with $m_{1}=1-\Phi_{1} / \Phi_{2}$, where $0 \leq m_{1} \leq 1$. Here $z_{0}$ is an arbitrary constant and the parameter $m_{1}$ is the modulus of the Jacobian elliptic function dn (see Refs. 11-13), which is periodic in $z$ with period $2^{1 / 4} \mathbf{K}\left(m_{1}\right) / \sqrt{\Phi_{2}}$, where $\mathbf{K}\left(m_{1}\right)$ is the complete elliptic integral of the first kind: $\mathbf{K}(m)=\int_{0}^{\pi / 2} d \theta / \sqrt{1-m_{1} \sin ^{2} \theta}$. In order to satisfy the periodical boundary conditions we find that the perimeter of the circle should be equal to an integer number $n$ of waves, from which we obtain

$$
a \sqrt{\Phi_{2}}=n 2^{1 / 4} \mathbf{K}\left(m_{1}\right), \quad n=1,2, \ldots .
$$

We also require that the wave functions are normalized $\int_{0}^{a} d z \phi_{1(2)}^{2}(z)=1$. Using this normalization condition and Eq. (2.13) we arrive at the relation

$$
\sqrt{2} \mathbf{K}\left(m_{1}\right) \mathbf{E}\left(m_{1}\right)=\frac{a}{n^{2}},
$$

where the complete elliptic integral of the second kind with modulus $m$ is defined by $\mathbf{E}(m)=$ $\int_{0}^{\pi / 2} d \theta \sqrt{1-m \sin ^{2} \theta}$. This equation determines $m_{1}$. In order to find the energy $\varepsilon_{0, n}^{(1)}=\sqrt{2}(B-A)$ we still need

$$
\begin{aligned}
B & =\int_{0}^{a} d z \phi_{1}^{4}(z)=\frac{2 n^{4} \mathbf{K}^{3}\left(m_{1}\right)}{a^{3}} \int_{0}^{\mathbf{K}\left(m_{1}\right)} d z \operatorname{dn}^{4}\left(z \mid m_{1}\right) \\
& =\frac{2 n^{4} \mathbf{K}^{3}\left(m_{1}\right)}{3 a^{3}}\left[2\left(2-m_{1}\right) \mathbf{E}\left(m_{1}\right)-\left(1-m_{1}\right) \mathbf{K}\left(m_{1}\right)\right]
\end{aligned}
$$

while $A$ takes the form

$$
A=\Phi_{2}\left(2-m_{1}\right)=\frac{\sqrt{2} n^{2} \mathbf{K}^{2}\left(m_{1}\right)}{a^{2}}\left(2-m_{1}\right) .
$$

With Eqs. (2.15) and (2.16) we arrive at the energy levels

$$
\varepsilon_{0, n}^{(1)}=\frac{1}{n^{2}} F_{1}\left(\frac{n^{2}}{a}\right)
$$

where $F_{1}(x)$ represents the universal function

$$
\begin{aligned}
F_{1}(x)= & -\frac{2}{3}\left[x \mathbf{K}\left(m_{1}\right)\right]^{2}\left[2-m_{1}\right. \\
& \left.+\sqrt{2} x \mathbf{K}^{2}\left(m_{1}\right)\left(1-m_{1}\right)\right] .
\end{aligned}
$$

For each $x$ we can solve Eq. (2.14) to find $m_{1}$ and calculate with this $m_{1}$ the function $F_{1}(x)$. Because $F_{1}(x)$ represents (up to a factor $1 / n^{2}$ ) the energies of all excited states we show in Fig. 1 the dependence of the parameter $m_{1}$ and the function $F_{1}$ on $x=n^{2} / a$. In the limiting case of a large circle $a \gg 1\left(m_{1} \rightarrow 1\right)$, we obtain from Eq. (2.14)

$$
m_{1}=1-16 e^{-\sqrt{2} / x}-128 e^{-2 \sqrt{2} / x} \frac{1-x^{2}}{x^{2}}+\cdots
$$

and

$\varepsilon_{0, n}^{(1)}=-\frac{1}{3 n^{2}}\left[1+24 e^{-\sqrt{2} a / n^{2}}+O\left(e^{-2 \sqrt{2} a / n^{2}}\right)\right]$.

In the opposite case when the radius of the circle decreases to zero, the left-hand side of Eq. (2.14) decreases monotonically and reaches its minimum value at $m_{1}=0$. 


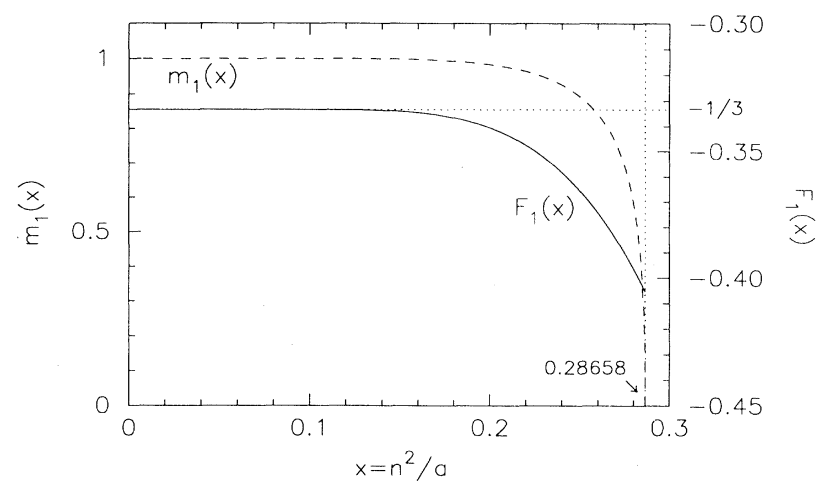

FIG. 1. The dependencies of the parameter $m_{1}$ and the function $F_{1}$ on $x=n^{2} / a$.

The parameter $x$ is then maximal. This implies that for each value of $n$ there exists a critical (minimal) value for the circle perimeter $a_{c}^{(n)}=n^{2} a_{c}$, where $a_{c}=\sqrt{2}(\pi / 2)^{2}$ at which the nontrivial real solution ceases to exist. At this critical value $a_{c}^{(n)}$ (or equivalently at $x_{c}=n^{2} / a_{c}^{(n)}=$ $\left.1 / a_{c}\right)$ the energy $\varepsilon_{0, n}^{(1)}$ becomes equal to $-(2 / \pi n)^{2}$ which coincides with the energy $\varepsilon_{0}^{(0)}$ of the constant wave function. In the vicinity of the critical point $\left(\delta a \approx a-n^{2} a_{c}\right)$ the energy behaves more precisely as follows

$$
\varepsilon_{0, n}^{(1)} \approx-\frac{1}{n^{2}}\left(\frac{2}{\pi}\right)^{2}\left[1-\frac{\Delta a}{n^{2} a_{c}}+O\left[(\Delta a)^{2}\right]\right] .
$$

Notice that not only do the curves $\varepsilon_{0}^{(0)}$ and $\varepsilon_{0, n}^{(1)}$ coincide at the critical points but also their slopes. The energy levels $\varepsilon_{0, n}^{(1)}$ versus the circle perimeter $a$ are plotted in Fig. 2 as solid curves. Those energy levels decrease indeed

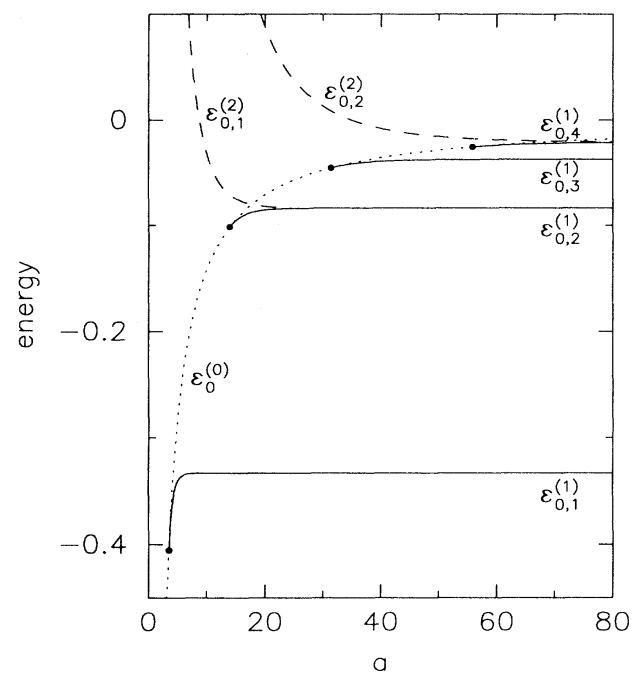

FIG. 2. Energy levels of a polaron on a circle vs the circle perimeter $a$ for the states with real wave functions. The energy of the constant solution corresponds to the dotted line. The solid lines indicate the energy levels of the symmetrical solutions (first set) and the dashed lines the asymmetrical ones (second set). with decreasing size of the circle until they reach their corresponding critical point.

\section{Second set of solutions}

Now we consider the case $\Phi_{1} \leq 0$, and rewrite Eq. (2.11) as follows:

$$
{\phi^{\prime}}^{2}(z)=2 \sqrt{2}\left[\phi^{2}(z)+\left|\Phi_{1}\right|\right]\left[\Phi_{2}-\phi^{2}(z)\right],
$$

from which we obtain $\Phi_{2} \geq 0,-\Phi_{2} \leq \phi^{2}(z) \leq \Phi_{2}$. The general solution to this equation is of the form ${ }^{10}$

$$
\phi_{2}(z)=\sqrt{\Phi_{2}} \operatorname{cn}\left[2^{3 / 4} \sqrt{\left|\Phi_{1}\right|+\Phi_{2}}\left(z-z_{0}\right) \mid m_{2}\right]
$$

with $m_{2}=\Phi_{2} /\left(\left|\Phi_{1}\right|+\Phi_{2}\right)$, where $0<m_{2} \leq 1$. This solution is periodic in $z$ with period $2^{5 / 4} \mathbf{K}\left(m_{2}\right) / \sqrt{\left|\Phi_{1}\right|+\Phi_{2}}$. From the boundary conditions it follows that

$$
a \sqrt{\left|\Phi_{1}\right|+\Phi_{2}}=2 n 2^{1 / 4} \mathbf{K}\left(m_{2}\right), \quad n=1,2, \ldots .
$$

These solutions coincide with the antisymmetrical solutions for the $1 \mathrm{D}$ system in an infinitely deep potential well of length $a$ as found in Ref. 10. The equation for the modulus $m_{2}$ of the elliptic function (2.23) is of the form

$$
\sqrt{2} \mathbf{K}^{2}\left(m_{2}\right)\left[\frac{\mathbf{E}\left(m_{2}\right)}{\mathbf{K}\left(m_{2}\right)}+m_{2}-1\right]=\frac{a}{n^{2}},
$$

and again the energies can be represented by a universal function:

$$
\varepsilon_{0, n}^{(2)}=\frac{1}{n^{2}} F_{2}\left(\frac{n^{2}}{a}\right)
$$

with

$$
\begin{aligned}
F_{2}(x)= & \frac{\sqrt{2}}{3}\left[x \mathbf{K}\left(m_{2}\right)\right]^{2} \\
& \times\left[2 m_{2}\left(1-m_{2}\right) x \mathbf{K}^{2}\left(m_{2}\right)-\sqrt{2}\left(2 m_{2}-1\right)\right] .
\end{aligned}
$$

For each $x$ we can solve Eq. (2.25) to find $m_{2}$ and calculate with this $m_{2}$ the function $F_{2}(x)$. Because also here $F_{2}(x)$ represents (up to a factor $1 / n^{2}$ ) the energies of all excited states we show in Fig. 3 the dependence of the parameter $m_{2}$ and the function $F_{2}$ on $x=n^{2} / a$. In the limiting case of a large circle $a \gg 1$, or equivalently $x \rightarrow 0$, we obtain

$$
\varepsilon_{0, n}^{(2)}=-\frac{1}{3(2 n)^{2}}\left[1-24 e^{-\sqrt{2} a /(2 n)^{2}}+O\left(e^{-2 \sqrt{2} a / n^{2}}\right)\right] .
$$

Thus, the energy levels tend to the same values as $\varepsilon_{0,2 n}^{(1)}$ (see Fig. 2). Note that for an infinitely deep potential well with length $a \gg 1$ we found in Ref. 10 the energy levels $\varepsilon_{n}=-1 / 3 n^{2}$ (cf. the first set of solutions).

In the opposite case when $a \ll 1$, we have $x \rightarrow \infty$, and from Eq. (2.25) 


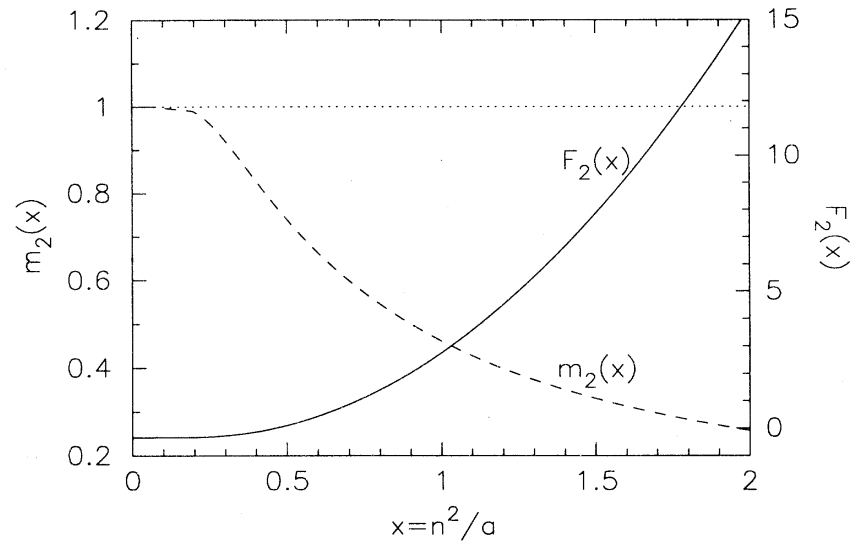

FIG. 3. The dependencies of the parameter $m_{2}$ and the function $F_{2}$ on $x=n^{2} / a$ (taken from Ref. 10).

$$
\varepsilon_{0, n}^{(2)}=\frac{\pi^{2} n^{2}}{2 a^{2}}-\frac{3 \sqrt{2}}{2 a}-\frac{1}{2 \pi^{2} n^{2}}+O(a)
$$

Note that reducing the perimeter of the circle will increase the confinement effects and we ultimately recover the energy levels of a particle in a box. The energies $\varepsilon_{0, n}^{(2)}$ vs $a$ are plotted in Fig. 2 by the dashed curves. In contrast to the first set of solutions we see that the energy of the antisymmetrical solutions is a decreasing function with increasing $a$.

The corresponding wave functions of both sets of real solutions are given in Fig. 4 for $z_{0}=0$ (which is a degeneracy parameter related to the position of the polaron). When the circle perimeter $a$ becomes infinitely large both wave functions (corresponding to $\varepsilon_{0,2}^{(1)}$ and $\varepsilon_{0,1}^{(2)}$ ) tend to a
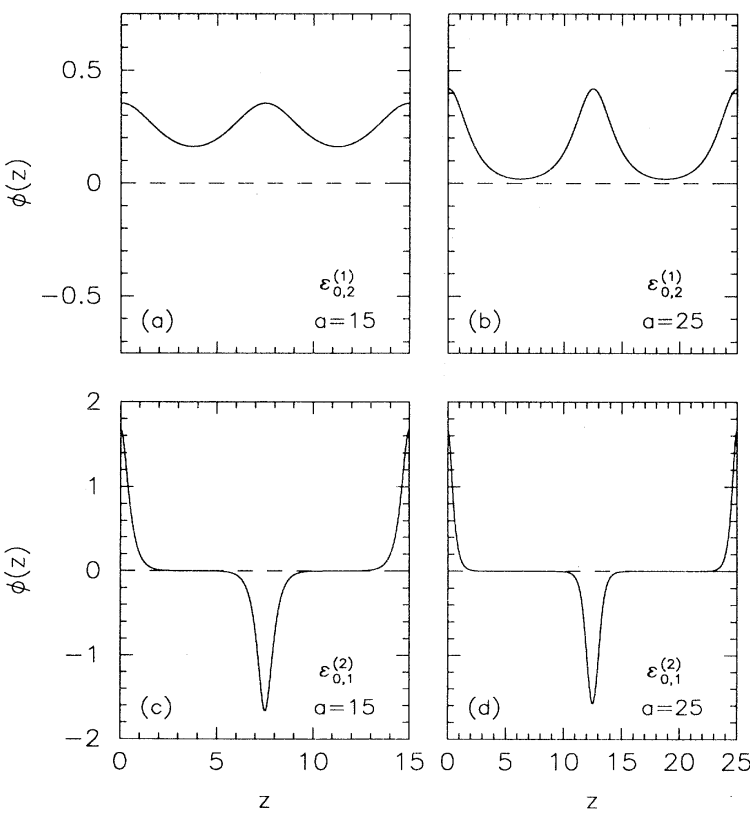

FIG. 4. The wave functions corresponding to the energies $\varepsilon_{0,2}^{(1)}$ and $\varepsilon_{0,1}^{(2)}$. wave function with infinitely separated peaks and attain the same energies (because the peaks only differ in sign). For higher excited states the number of peaks in the wave function increases; e.g., the number of peaks will be $n$ for the wave function corresponding to $\varepsilon_{0, n}^{(1)}$ and $2 n$ for the wave function corresponding to $\varepsilon_{0, n}^{(2)}$.

Note that solutions to the linear Schrödinger equation are usually classified by the number of zeros in the wave function, e.g., a solution $\phi_{n}$ has $n-1$ zeros. At the same time the probability distribution $\left|\phi_{n}\right|^{2}$ of this wave function has $n$ peaks. The present analysis indicates that in nonlinear physics there exist wave functions without zero points [see Fig. 4(a)] and wave functions with large areas, which are zero [see Fig. 4(d)]. Therefore, in the present case the classification of excited states can only be done by counting the number of peaks in the probability distribution which seems to be more general than the classification based on the counting of zero points.

\section{COMPLEX SOLUTIONS}

In the present section we will search for a solution to Eq. (2.5) of the form

$$
\psi(z)=\phi(z) e^{i \chi(z)},
$$

where the amplitude $\phi(z)$ and phase $\chi(z)$ are real functions of the coordinate $z$. Inserting Eq. (3.1) into Eq. (2.5) we arrive at the coupled set of equations

$$
\begin{gathered}
-\frac{1}{2} \phi^{\prime \prime}(z)+\left[\sqrt{2} B-2 \sqrt{2} \phi^{2}(z)+\frac{1}{2} \chi^{\prime 2}(z)-\varepsilon\right] \phi(z)=0 \\
\chi^{\prime \prime}(z) \phi(z)+2 \chi^{\prime}(z) \phi^{\prime}(z)=0
\end{gathered}
$$

The general solution to Eq. (3.2b) can be represented as

$$
\chi(z)=\chi(0)+Q \int_{0}^{z} \frac{d z^{\prime}}{\phi^{2}\left(z^{\prime}\right)},
$$

where $Q$ is an arbitrary real constant. After inserting this solution into Eq. (3.2a) we arrive at the nonlinear effective Schrödinger equation for the amplitude $\phi(z)$

$$
-\frac{1}{2} \phi^{\prime \prime}(z)+\sqrt{2} A \phi(z)-2 \sqrt{2} \phi^{3}(z)+\frac{Q^{2}}{2 \phi^{3}(z)}=0,
$$

with $A=B-\varepsilon / \sqrt{2}$. For $Q=0$ we arrive at the previous set of real solutions. For the external potentials considered in Ref. 10 the set of real solutions are the only existing solutions to Eq. (2.5). Note that if on an infinite axis both the potential and the amplitude $\phi(z)$ vanish at infinity it follows from Eq. (3.4) that $Q=0$ and consequently no complex solutions will exist. The same is true if the wave function has zeros on a finite segment. Indeed, if $\phi(z) \approx c\left(z-z_{0}\right)^{\beta}$ at some point $z_{0}$ then $\phi^{\prime \prime}(z) \approx c \beta(\beta-1)\left(z-z_{0}\right)^{\beta-2}$ and the term with the second derivative has to cancel with the last term of Eq. (3.4) from which we ob- 
tain $c \beta(\beta-1) /\left(z-z_{0}\right)^{2-\beta}=Q^{2} /\left[c^{3}\left(z-z_{0}\right)^{3 \beta}\right]$. Consequently we find $\beta=1 / 2$ and $Q^{2}=-c^{4} / 4$ which is in contradiction with our requirement that $Q$ and $c$ are real parameters.

Here we will show that on a circle complex solutions do exist and can be classified by an integer number $l_{z}$, which is the angular momentum. Periodic boundary conditions imply for the phase $\chi(a)=\chi(0)+2 \pi l_{z}, l_{z}=$ $0, \pm 1, \pm 2, \ldots$, which results in

$$
Q=2 \pi l_{z}\left(\int_{0}^{a} \frac{d z^{\prime}}{\phi^{2}\left(z^{\prime}\right)}\right)^{-1}
$$

The most simple solution is found for a constant amplitude $\phi(z)=1 / \sqrt{a}$. In this case $B=1 / a, Q=2 \pi l_{z} / a^{2}$ and for the phase we obtain the conventional result of a quantum-mechanical particle on a circle: $\chi(z)=$ $\chi(0)+2 \pi l_{z} z / a$. The corresponding energy levels are described by the formula

$$
\varepsilon_{l_{z}}^{(0)}=-\frac{\sqrt{2}}{a}+\left(\frac{2 \pi}{a}\right)^{2} \frac{l_{z}^{2}}{2} .
$$

For $l_{z}=0$ we arrive at the energy (2.9). The second term in Eq. (3.6) is the conventional centrifugal part of the kinetic energy. The energy levels $\varepsilon_{l_{z}}^{(0)}$ for $l_{z}=0,1,2,3$ are shown in Fig. 5. The energy levels for $l_{z} \geq 1$ attain a local minimum $\varepsilon_{l_{z}, \min }^{(0)}=-1 /\left(2 \pi l_{z}\right)^{2}$ at $a=2 \sqrt{2}\left(\pi l_{z}\right)^{2}$ and tend to their limiting values at large $a$ from below. The curve for $l_{z}=0$ that corresponds to the constant wave function of the real solutions (see also Fig. 2) obtains a lower energy for decreasing $a$. For $l_{z} \geq 1$, however, we see clearly the increase of the energy for small $a$ due to the centrifugal contribution which becomes more important the larger $l_{z}$ is and the smaller $a$ is. The corresponding wave functions are $(1 / \sqrt{a}) e^{2 \pi i l_{z} z / a}$ up to an unimportant constant phase factor.

In the remainder of this section we will search for other

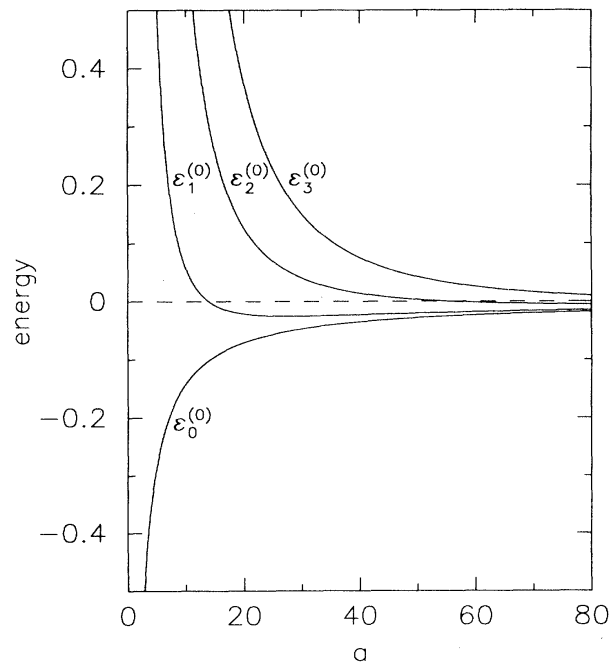

FIG. 5. Energies of a polaron on a circle vs the scaled circle perimeter for the states with complex wave functions. complex solutions. We use the first integral of Eq. (3.4)

$$
-\frac{1}{4} \phi^{\prime 2}(z)+\frac{\phi^{2}(z)}{\sqrt{2}}\left[A-\phi^{2}(z)\right]-\frac{Q^{2}}{4 \phi^{2}(z)}=C,
$$

where $C$ is an integration constant. Introducing the notations $\Phi(z)=\phi^{2}(z), A=\Phi_{\max }+$ $\Phi_{\min }-\Phi_{0}, \sqrt{2} C=\Phi_{\max } \Phi_{\min }-\Phi_{0}\left(\Phi_{\max }+\Phi_{\min }\right)$, and $Q^{2}=2 \sqrt{2} \Phi_{\max } \Phi_{\min } \Phi_{0}$, where all parameters $\left(\Phi_{0}, \Phi_{\min }, \Phi_{\max }\right)$ are positive and $\Phi_{\min } \leq \Phi_{\max }$, we arrive at the equation

$$
\Phi^{\prime 2}(z)=8 \sqrt{2}\left[\Phi(z)+\Phi_{0}\right]\left[\Phi(z)-\Phi_{\min }\right]\left[\Phi_{\max }-\Phi(z)\right] .
$$

It follows that (1) solutions can only exist if $Q^{2}\left(l_{z}^{2}\right)$ does not exceed some maximal value, and (2) $0 \leq \Phi_{\min } \leq$ $\phi^{2}(z) \leq \Phi_{\max }$, where $\phi^{2}(z)$ does not reach zero when $Q^{2} \neq 0$, which implies that $\phi(z)$ has a constant sign and no nodes. Equation (3.8) can be integrated and results in

$$
\phi^{2}(z)=\frac{\Phi_{\min }+m \Phi_{0} \mathrm{sn}^{2}(u \mid m)}{1-m \mathrm{sn}^{2}(u \mid m)}
$$

where $u=2^{3 / 4} \sqrt{\Phi_{\max }+\Phi_{0}}\left(z-z_{0}\right)$ and $m=$ $\left(\Phi_{\max }-\Phi_{\min }\right) /\left(\Phi_{\max }+\Phi_{0}\right)$. Equivalently, Eq. (3.9) can be written as follows:

$$
\phi^{2}(z)=\Phi_{\min }+\left(\Phi_{\max }-\Phi_{\min }\right) \mathrm{cn}^{2}(u \mid m),
$$

where only the constant term $z_{0}$ should be changed in the definition of $u$. Note that at $Q=0$ we arrive either at solution (2.12) [i.e., $\phi_{1}(z)$ ] with $\Phi_{\max }=\Phi_{2}, \Phi_{\min }=\Phi_{1}$ and $\Phi_{0}=0$ or at solution (2.23) [i.e., $\phi_{2}(z)$ ] with $\Phi_{\max }=$ $\Phi_{2}, \Phi_{\min }=0$ and $\Phi_{0}=\left|\Phi_{1}\right|$.

The periodicity in $u$ on the circle implies that there should be an integer number $(n)$ of waves on the perimeter of the circle. Because the period in $u$ equals $2 \mathbf{K}(m)$ one has

$$
2^{3 / 4} \sqrt{\Phi_{\max }+\Phi_{0}} a=2 n \mathbf{K}(m) .
$$

From the periodicity condition (3.11), the normalization condition and the definition of $m$ all the parameters $\left(\Phi_{0}, \Phi_{\min }\right.$, and $\left.\Phi_{\max }\right)$ can be determined. The normalization condition $\int_{0}^{a} d z \phi^{2}(z)=1$ leads to

$$
\Phi_{\min }=\frac{1}{a}\{1-\sqrt{2} x \mathbf{K}(m)[\mathbf{E}(m)-(1-m) \mathbf{K}(m)]\},
$$

the definition of $m$ gives

$$
\Phi_{\max }=\frac{1}{a}\{1+\sqrt{2} x \mathbf{K}(m)[\mathbf{K}(m)-\mathbf{E}(m)]\}
$$

and finally from the periodicity condition (3.11) we obtain

$$
\Phi_{0}=\frac{1}{a}\{-1+\sqrt{2} x \mathbf{K}(m) \mathbf{E}(m)\}
$$


The parameter $m$ itself can be obtained combining Eq. (3.5) with its parametrized form. This leads to the equation

$$
2 \pi \sqrt{y}=2^{3 / 4} \sqrt{\left(\Phi_{0} a\right)(1-\gamma)} \frac{\boldsymbol{\Pi}(\gamma, m)}{\mathbf{K}(m)},
$$

where

$$
\Pi(\gamma, m)=\int_{0}^{\pi / 2} \frac{d \phi}{\left[1-\gamma \sin ^{2} \phi\right] \sqrt{1-m \sin ^{2} \phi}},
$$

which is the complete elliptic function of the third kind, with $y=l_{z}^{2} / a$ and $\gamma=\sqrt{2} x m \mathbf{K}^{2}(m) /\{1$ $+\sqrt{2} x \mathbf{K}(m)[\mathbf{K}(m)-\mathbf{E}(m)]\}$. From Eq. (3.15) one can determine $m$ for given quantum numbers $n$ and $l_{z}$.

Also the energy $\varepsilon=\sqrt{2}(B-A)$ can be determined as a function of $m$. The parameter $A$ is given by

$$
A=\frac{1}{a}\left\{3-3 \sqrt{2} x \mathbf{K}(m) \mathbf{E}(m)+\sqrt{2}(2-m) x \mathbf{K}^{2}(m)\right\},
$$

and $B=\int_{0}^{a} d z|\phi(z)|^{4}$ has the form

$$
\begin{aligned}
B= & \frac{1}{a}\left\{1+\frac{2}{3} x^{2} \mathbf{K}^{2}(m)\left[-3 \mathbf{E}^{2}(m)+2(2-m) \mathbf{E}(m) \mathbf{K}(m)\right.\right. \\
& \left.\left.-(1-m) \mathbf{K}^{2}(m)\right]\right\} .
\end{aligned}
$$

From Eqs. (3.17) and (3.18) the energy levels corresponding to the complex solutions can be calculated

$$
\varepsilon_{l_{z}, n}=\left(1 / n^{2}\right) F_{12}(x, y)
$$

with

$$
\begin{aligned}
F_{12}(x, y)= & \sqrt{2} x\{-2+\sqrt{2} x \mathbf{K}(m)[3 \mathbf{E}(m) \\
& -(2-m) \mathbf{K}(m)]+\frac{2}{3} x^{2} \mathbf{K}^{2}(m)\left[-3 \mathbf{E}^{2}(m)\right. \\
& \left.\left.+2(2-m) \mathbf{E}(m) \mathbf{K}(m)-(1-m) \mathbf{K}^{2}(m)\right]\right\},
\end{aligned}
$$

where $m=m(y)$ is obtained by solving Eq. (3.15).

Numerical calculations indicated, however, that Eq. (3.15) has no solutions for $l_{z} \geq 1$. Therefore, we arrive at the important conclusion that the only complex solutions are $(1 / \sqrt{a}) e^{2 \pi i l_{z} z / a}$. Only for a circle surrounding a magnetic flux more complicate complex solutions exist. This case will be discussed in a subsequent paper.

\section{CONCLUSIONS}

In this paper the nonlinear effective Schrödinger equation was solved on an infinitesimal thin ring or circle. Exact real and complex solutions are found together with their corresponding energies. In the case of real symmetrical solutions we found critical values $a_{c}^{(n)}$ of the circle perimeter such that for $a<a_{c}^{(n)}$ the solution no longer exists. Therefore, the larger the circle perimeter the more excited states with real wave functions exist. The complex wave functions correspond to energy levels with finite angular momentum $l_{z}$ and are of the form $\Psi(z)=(1 / \sqrt{a}) e^{2 \pi i l_{z} z / a}$. Here we found that counting the number of peaks in the wave function can be used to classify the quantum number of the excited state. This is in contrast with linear problems where usually the number of nodes in the wave function is used.

If we compare the above solutions with the eigenstates from which, e.g., Büttiker et al. ${ }^{18}$ started, we see that the additional real solutions, we found, could have some significance. Therefore, the method to treat persistent currents (cf. Ref. 1) should start from the nonlinear effective Schrödinger equation, e.g., by applying flux-modified boundary conditions (for more details about this method see Refs. 1, 19, and 20).

\section{ACKNOWLEDGMENTS}

We are indebted to V. M. Fomin, Yu. M. Kagan, and W. van Haeringen for stimulating discussions. M.A.S. is grateful to the University of Antwerp (UIA) for the support and kind hospitality during his visits to Belgium. P.V. thanks the NFWO and the Joint Institute for Nuclear Research for the support of his visit to Dubna. F.M.P. is supported by the Belgian National Science Foundation (NFWO). This work is partly performed in the framework of "Diensten voor de Programmatie van het Wetenschapsbeleid" (Belgium) under contract No. IT/SC/24, the FKFO project 2.0093.91, and the Human Capital and Mobility Program "Polarons, bipolarons and excitons. Properties and occurrence in new materials" (C.E.C. No. CHRX-CT93-0124).

\footnotetext{
* Permanent address: N.N. Bogoliuboy Laboratory of Theoretical Physics, Joint Institute for Nuclear Research, 141980 Dubna, Russia. Electronic address: smond@thsun1.jinr.dubna.su

† Electronic address: vansant@uia.ua.ac.be

‡ Electronic address: peeters@uia.ua.ac.be

$\S$ Also at Universiteit Antwerpen (RUCA), Groenenborgerlaan 171, 2020 Antwerpen, Belgium and Technische Universiteit Eindhoven, P.O. Box 513, 5600 MB Eindhoven, Netherlands. Electronic address: devreese@uia.ua.ac.be

${ }^{1}$ H. F. Cheung, Y. Gefen, E. K. Riedel, and W. H. Shih, Phys. Rev. B 37, 6050 (1988).
}

${ }^{2}$ M. Büttiker, Y. Imry, and R. Landauer. Phys. Lett. 96A, 365 (1983)

${ }^{3}$ M. Büttiker, Phys. Rev. B 32, 1846 (1985).

${ }^{4}$ Various contributions in Physics of Optical Ring Gyros, edited by S. F. Jacobs, M. Sargent III, M. O. Scully, J. Simpson, V. Sanders, and J. E. Killpatrick (SPIE, Bellingham, WA, 1984), Vol. 487.

${ }^{5}$ P. Lett, W. Christian, S. Singh, and L. Mandel, Phys. Rev. Lett. 47, 1892 (1981); L. Mandel, R. Roy, and S. Singh, in Optical Bistability, edited by C. M. Bowden, M. Ciftan, and Th. R. Roble (Plenum, New York, 1981).

${ }^{6}$ R. J. C. Spreeuw, J. P. Woerdman, and D. Lenstra, Phys. 
Rev. Lett. 61, 318 (1988).

${ }^{7}$ D. Lenstra and S. H. M. Geurten, Opt. Commun. 75, 63 (1990).

8 Analogies in Optics and Micro-Electronics, edited by W. van Haeringen and D. Lenstra (North-Holland, Amsterdam, 1991).

${ }^{9}$ A. C. Scott, F. Y. F. Chu, and D. W. McLaughlin, Proc. IEEE 61, 1443 (1973).

${ }^{10}$ P. Vansant, M. A. Smondyrev, F. M. Peeters, and J. T. Devreese, Z. Phys. B (to be published).

${ }^{11}$ Handbook on Mathematical Functions, National Bureau of Standards Applied Mathematics Series No. 55, edited by M. Abramowitz and I. A. Stegun (U.S. GPO, Washington, D.C., 1964), Chap. 16.

${ }^{12}$ I. S. Gradshteyn and I. M. Ryzhik, Tables of Integrals,
Sums, Series and Products (Academic, New York, 1965).

${ }^{13}$ A. S. Davydov, Solitons in Molecular Systems, 2nd ed. (Naukova Dumka, Kiev, 1988) [see also the English translation of the 1st edition (Reidel, Dordrecht, Holland, 1985)].

${ }^{14}$ E.P. Gross, Ann. Phys. (N.Y.) 99, 1 (1976).

${ }^{15}$ U. Mansmann, Stochastics Stochastics Rep. 34, 93 (1991).

${ }^{16}$ F. M. Peeters, X. Wu, and J. T. Devreese, Phys. Rev. B 33, 3926 (1986).

${ }^{17}$ F. M. Peeters and M. A. Smondyrev, Phys. Rev. B 43, 4920 (1991).

${ }^{18}$ M. Büttiker, Y. Imry, R. Landauer, and S. Pinhas, Phys. Rev. B 31, 6207 (1985).

${ }^{19}$ N. Byers and C. N. Yang, Phys. Rev. Lett. 7, 46 (1961).

${ }^{20}$ F. Bloch, Phys. Rev. B 2, 109 (1970). 